\title{
El Carcinoma de Vulva en el Hospital Universitario
}

\author{
PERIODO DE 1964 - 1972
}

Dres. : Jorge E. Zúñiga C. * y Fortunato Aljure **

\section{SINTESIS}

El carcinoma de la vulva representa el $5 \%$ de todos los tumores malignos del aparato genital femenino. Se sabe que es una enfermedad propia de mujeres mayores de 60 años y más de la raza negra.

En este estudio se presentan los resultados obtenidos en 17 casos tratados en el per íodo 1964-1972 en el Hospital Universitario de Cali - Col - S.A.

En este grupo de pacientes se analizan: edad, enfermedades asociadas, frecuencia de aparición del tumor según sitios anatómicos, tipo histológico, demora en el diagnóstico, tipos de tratamiento y la supervivencia de las pacientes que tuvieron seguimiento adecuado.

\section{MATERIAL}

Los 17 casos de carcinoma invasivo de vulva corresponden a pacientes de la consulta externa del Hospital Universitario de Cali. El seguimiento ha sido bastante dificultoso porque las pacientes una vez notan mejoría no regresan para control. Del grupo total de 17 pacientes del estudio 12 , ó sea el $(70 \%)$, tenían más de

* Profesor Asociado Depto. Obst y Ginec.

* Profesor de Cirugía Univalle
50 años de edad. La mayor parte del grupo eran pacientes grandes multíparas con 6 ó más embarazos; sólo 3 casos en nulíparas, el $70 \%$ (12 casos) de un nivel socio-económico muy bajo.

\section{PACIENTES POR GRUPOS DE EDAD}

\begin{tabular}{|c|c|c|c|c|}
\hline $30-39$ & $40-49$ & $50-59$ & $60-69$ & $70+$ \\
\hline 3 & 2 & 4 & 6 & 2 \\
\hline
\end{tabular}

SE PRESENTAN 12 CASOS $(70 \%)$ POR ENCIMA DE LOS 50 AÑOS.

Relación con enfermedades venéreas

En un caso se tuvo el antecedente de sífilis, en 2 casos historia de condiloma acuminado, en 4 pacientes había historia de intensa promiscuidad sexual.

PACIENTES SEGUN GRAVIDEZ Y PARIDAD

\begin{tabular}{|c|c|c|c|c|}
\hline $1-3$ & $4-6$ & $6+$ & NULIPARAS & SIN DATO \\
\hline 4 & 2 & 6 & 3 & 2 \\
\hline
\end{tabular}

LA MAYORIA DE LAS PACIENTES FUERON GRANDES MULTIPARAS, CON MAS DE SEIS EMBARAZOS $Y$ PARTOS. 
FACTOR SOCIOECONOMICO

\begin{tabular}{|c|c|c|}
\hline BAJO & MEDIO & ALTO \\
\hline 12 & 3 & $2^{*}$ \\
\hline
\end{tabular}

LA MAYORIA DE LAS PACIENTES DE UN MEDIO SOCIOECONOMICO MUY BAJO.

* CA basocelular

CA GLANDULAS APOCRINAS

\section{Tipo histológico}

Carcinoma epidermoide en 14 casos. Melanoma un caso.

Carcinoma de glándula de Bartholin un caso.

Carcinoma basocelular un caso.

Raza

El mayor número de pacientes de raza mestiza.

Tiempo entre la iniciación de los síntomas y la primera consulta

La mayoría de las pacientes tardaron entre 6 meses a un año para hacer la con- sulta una vez iniciados los síntomas. EI síntoma principal fue la aparición de una lesión ulcerada pruriginosa, con evolución posterior a masa tumoral.

En 6 pacientes la enfermedad se asociaba a obesidad extrema.

RELACION CON

ENFERMEDADES VENEREAS

\begin{tabular}{|c|c|c|}
\hline CONDILOMAS & LUES & PROSTITUCION \\
\hline 2 & 1 & $4 *$ \\
\hline
\end{tabular}

EN ALGUNOS CASOS POR REVISION CUIDADOSA DE LAS HISTORIAS CLINICAS SE ENCONTRO EL ANTECEDENTE DE ENFERMEDAD VENEREA.

EN 4 CASOS PROMISCUIDAD SEXUAL.

* del total, 3 corresponden al grupo de $30-40$ AÑOS.

PACIENTES POR GRUPOS DE RAZA

\begin{tabular}{|c|c|c|c|}
\hline MESTIZA & NEGRA & BLANCA & SIN \\
\hline 8 & 6 & 1 & 2 \\
\hline
\end{tabular}

LA MAYORIA DE LAS PACIENTES DE LA RAZA MESTIZA, SEGUIDA DE LA RAZA NE

DIAGNOSTICO HISTOLOGICO (BIOPSIAS)

\begin{tabular}{|c|c|c|c|}
\hline CA EPIDERMOIDE & MELANOMA & $\begin{array}{l}\text { ADENOCARCINOMA DE } \\
\text { GLANDULAS ENDOCRINAS }\end{array}$ & $\begin{array}{l}\text { CA BASO- } \\
\text { CELULAR }\end{array}$ \\
\hline 14 & 1 & 1 & 1 \\
\hline
\end{tabular}

\section{LA GRAN MAYORIA DE LAS PACIENTES CON CARCINOMA} EPIDERMOIDE. 
LOCALIZACION TUMOR PRIMARIO

\begin{tabular}{|c|c|c|c|c|c|c|}
\hline \multirow{2}{*}{ LABIOS } & MENORES & \multicolumn{2}{|c|}{ LABIOS MAYORES } & \multirow{2}{*}{ CLITORIS } & LABIOS MAYORES & \multirow{2}{*}{ LIFUSO * } \\
\cline { 1 - 3 } & 1 & $D$ & 1 & & Y MENORES & \\
\hline 1 & 1 & 4 & 4 & 2 & 4 & 1 \\
\hline
\end{tabular}

GANGLIOS CLINICAMENTE SIGNIFICATIYOS EN 5 CASOS.

* carcinoma epidermoide in situ de vulva-Vagina-cervix.

TRATAMIENTO QUIRURGICO

\begin{tabular}{|l|c|}
\hline VULVECTOMIA SIMPLE & 3 CASOS \\
\hline VULVECTOMIA RADICAL & 2 CASOS \\
\hline $\begin{array}{l}\text { VULVECTOMIA RADICAL CON VACIAMIENTO } \\
\text { BILATERAL SUPERFICIAL Y PROFUNDO }\end{array}$ & 5 CASOS \\
\hline $\begin{array}{l}\text { VULVECTOMIA RADICAL CON VACIAMIENTO INGUINAL } \\
\text { IZQUIERDO SUPERFICIAL Y PROFUNDO }\end{array}$ & 1 CASO \\
\hline $\begin{array}{l}\text { VULVECTOMIA RADICAL CON VACIAMIENTO INGUINAL } \\
\text { DERECHO SUPERFICIAL Y PROFUNDO }\end{array}$ & 2 CASOS \\
\hline $\begin{array}{l}\text { VULVECTOMIA RADICAL CON VACIAMIENTO INGUINAL } \\
\text { SUPERFICIAL Y PROFUNDO IZQUIERDO Y VACIA- } \\
\text { MIENTO INGUINAL SUPERFICIAL DERECHO }\end{array}$ & 3 CASOS \\
\hline $\begin{array}{l}\text { VULVECTOMIA RADICAL Y VACIAMIENTO } \\
\text { INGUINAL SUPERFICIAL BILATERAL }\end{array}$ & 2 CASOS \\
\hline \multicolumn{1}{|c|}{ TOTAL } & 18 CASOS \\
\hline
\end{tabular}

MORTALIDAD OPERATORIA: O CASOS

* paciente con neVus en pantalon y neurofibromatosis 
ANALISIS 13 VACIAMIENTOS GANGLIONARES INGUINALES

\begin{tabular}{|c|c|c|c|c|c|c|}
\hline \multicolumn{6}{|c|}{$\begin{array}{l}\text { GANGLIOS + } \\
\text { TOTAL } 9\end{array}$} & $\begin{array}{l}\text { GANGLIOS- } \\
\text { TOTAL } 4 \\
\end{array}$ \\
\hline \multicolumn{2}{|c|}{ DERECHOS } & \multicolumn{2}{|c|}{ IZQUIERDOS } & \multicolumn{2}{|c|}{ BILATERALES } & \\
\hline SUPERFICIAL & PROFUNDO & SUPERFICIAL & PROFUNDO & SUPERFICIAL & PROFUNDO & \\
\hline 3 & 0 & 7 & 2 & 2 & 0 & 4 \\
\hline
\end{tabular}

Sitio primario de la lesión tumoral

En los labios mayores en 8 casos:

4 en el izquierdo, y 4 en el derecho.

En labios menores dos casos:

1 en el izquierdo y otro en el derecho.

En clitoris dos casos:

Ganglios palpables sospechosos de metástasis en 5 casos.

\section{Conducta :}

Se practicó vulvectomía simple en 3 casos, radical en 14 casos, radical con vaciamiento bilateral superficial y profundo en 5 casos, radical con vaciamiento inguinal superficial y profundo izquierdo en un caso.
Vulvectomía radical con vaciamiento inguinal superficial y profundo derecho en 2 casos.

EVOLUCION DE LOS SINTOMAS A LA PRIMERA CONSULTA

\begin{tabular}{|c|c|c|c|c|}
\hline $\begin{array}{c}0-6 \\
\text { MESES }\end{array}$ & $\begin{array}{c}6-12 \\
\text { MESES }\end{array}$ & $\begin{array}{c}1-2 \\
\text { AÑOS }\end{array}$ & $\begin{array}{c}2 \text { AÑOS } \\
\text { Y }\end{array}$ & $\begin{array}{l}\text { SIN } \\
\text { DATO }\end{array}$ \\
\hline 5 & 8 & - & 2 & 2 \\
\hline
\end{tabular}

LA MAYORIA DE LAS PACIENTES PRESENTARON SINTOMAS DE 6 A I2 MESE ANTES DE HACER LA CONSULTA.

SINTOMAS PRINCIPALES

\begin{tabular}{|c|c|c|c|c|c|c|}
\hline $\begin{array}{c}\text { MASA } \\
\text { INGUINAL }\end{array}$ & DOLOR & FLUJO & PRURITO & ULCERACION & TUMOR $^{*}$ & $\begin{array}{c}\text { ASINTO- } \\
\text { MATICAS }\end{array}$ \\
\hline 2 & 2 & 3 & 6 & 10 & 5 & 2 \\
\hline
\end{tabular}

SE ASOCIO LA OBESIDAD EN 6 CASOS

EN UN CASO TUBERCULOSIS PULMONAR. NO SE REGISTRO DIABETES ASOCIADA.

* tumor eN labios. 
Vulvectomía radical con vaciamiento inguinal superficial y profundo izquierdo y superficial derecho en 3 casos.

Vulvectomía radical y vaciamiento inguinal superficial bilateral en 2 casos.

\section{RESULTADOS DE TRATAMIENTO EN 10 PACIENTES CON CONTROLES DE 5 O MAS AÑOS- 1964 - 1968}

\begin{tabular}{|c|c|c|c|c|c|}
\hline \multirow{2}{*}{$\begin{array}{l}\text { CASO } \\
\text { No. }\end{array}$} & \multirow{2}{*}{$\begin{array}{l}\text { CURACION } \\
\text { NEE(ANOS) }\end{array}$} & \multirow{2}{*}{$\begin{array}{c}\text { SOBREVIDA } \\
\text { (ANOS) }\end{array}$} & \multicolumn{3}{|c|}{ GANGLIOS } \\
\hline & & & + & - & SIN DATO \\
\hline 1 & 9 & & & - & \\
\hline 2 & 6 & & & & SD \\
\hline 6 & 9 & & & - & \\
\hline 9 & & $3 *$ & + & & \\
\hline 11 & 7 & & + & & \\
\hline 13 & 5 & & & - & \\
\hline 14 & 7 & & + & & \\
\hline 15 & 5 & & & & SD \\
\hline $16 *$ & (S.C) & & + & & \\
\hline 17 & (S.C) & & + & & \\
\hline & & TOTAL & 5 & (3) & 2 \\
\hline & & GRAN TOTAL & & 10 & \\
\hline
\end{tabular}

* muRIo. masa pelvica IZQUIERDa

DIAGNOSTICO TARDIO. MASA INGUINAL ULCERADA ** paciente que consulto por recurRencia local CURACION POR ENCIMA DE 5 AÑOS -.... 7 CASOS SIN CONTROL $\ldots \ldots$ CASOS MORTALIDAD POR CANCER -....... I CASO

No hubo mortalidad operatoria. En 13 casos de vaciamientos ganglionares superficiales y profundos, se encontraron metástasis en 9 casos: en 3 en el lado derecho, en 6 en el izquierdo, en 2 metástasis ganglionares bilaterales, 4 casos sin metástasis.

\section{Supervivencia}

Dos pacientes con controles a 9 años, dos a 7, una a 6 , dos a 5 , sin evidencia de enfermedad. Cuatro pacientes tuvieron controles entre 1 y 3 años sin evidencia de enfermedad. Se perdieron del control. Controles a más de 5 años bien, 7 pacientes $(50 \%)$.

La paciente del melanoma murió a los $21 / 2$ años por metástasis cerebrales. Dos
SOBREVIDA 1964 - 1972

\begin{tabular}{|c|c|c|c|c|}
\hline & $\begin{array}{c}\text { SOBREVIDA } \\
\text { TIEMPO }\end{array}$ & $\begin{array}{l}\text { CON METASTASIS } \\
\text { A LA CIRUGIA }\end{array}$ & $\begin{array}{l}\text { SIN METASTASIS } \\
\text { A LA CIRUGIA }\end{array}$ & SIN DATO \\
\hline 1 & 8 AÑOS & & 1 & \\
\hline 2 & 6 AÑNOS & & & \\
\hline 3 & I AÑO & $\begin{array}{ll}3 & \text { NEE } \\
\end{array}$ & & \\
\hline 4 & II/2 AÑOS & $4 \quad \mathrm{NEE}$ & & \\
\hline 5 & 2 AÑOS & & $\begin{array}{ll}5 & \mathrm{NEE} \\
\end{array}$ & \\
\hline 6 & 8 AÑOS & & \begin{tabular}{|ll}
6 & NEE
\end{tabular} & \\
\hline $7+$ & 8 MESES & $\begin{array}{l}7 \text { MASIVO } \\
\text { PELVIS }\end{array}$ & & \\
\hline 8 & 3 AÑOS & & 8 CA IN SITU & \\
\hline $9+$ & 8 MESES & $\begin{array}{l}9 \text { MASIVO } \\
\text { PELVIS }\end{array}$ & & $\begin{array}{l}\text { NO RECIDI } \\
\text { VA LOCAL }\end{array}$ \\
\hline $10+$ & I AÑO & & & 10 \\
\hline 11 & 7 AÑOS & II NEE & & \\
\hline $12+$ & 2 1/2AN̄OS & $\begin{array}{l}12 \text { MELANOMA } \\
\text { (METASTAS }\end{array}$ & IS (EEREBRAL) & \\
\hline 13 & 5 AÑOS & & 13 & \\
\hline 14 & 7 AÑOS & $\begin{array}{l}14 \mathrm{NEE} \\
\text { (ADENOCARCIN }\end{array}$ & OMA G. APOCRIN & \\
\hline 15 & 5 AÑOS & & $15 \begin{array}{c}\text { (CA } \\
\text { CELULASO- }\end{array}$ & \\
\hline 16 & $?$ & 16 & & \\
\hline 17 & $?$ & 17 & & \\
\hline
\end{tabular}

pacientes murieron a los 8 meses por metástasis difusas a la pelvis.

Es de anotar que todo este grupo de pacientes consultó ya con lesiones tumorales mayores de $3 \mathrm{cmts}$. y ganglios regionales palpables.

\section{RESUMEN}

Se presenta un estudio de 17 casos de cáncer de vulva en el Hospital Universitario del Valle en el período 1964-1972.

El $70 \%$ de las pacientes tenían más de 50 años; la mayoría grandes multíparas de un nivel socio-económico bajo; la relación con enfermedad venérea en este grupo fue muy pobre, sólo 3 casos. En 14 casos el cáncer fue epidermoide. En la mayoría de los casos hubo demora en el diagnóstico, por consulta tardía. El síntoma principal fue la úlcera pruriginosa, la obesidad se asoció en 6 casos, en 10 casos se inició la enfermedad en los labios mayores y menores, 2 casos en el clítoris.

El tratamiento fue vulvectomía radical en 14 casos y vulvectomía total en 3 . Su- 
pervivencia mayor de 5 años: 7 casos $(50 \%)$.

Los controles periódicos fueron difí. ciles por falta de colaboración de las pacientes. La mayoría de los casos eran estadios clínicos II y III.

Es necesario un diagnóstico temprano para mejorar la supervivencia en este grupo de pacientes; una vez iniciados los sintomas hubo demora hasta de un año para hacer el diagnóstico y el tratamiento adecuados.

Se conoce la relación entre el tamaño de la lesión y la posibilidad de metástasis; la mayoría de las pacientes tenían la enfermedad muy avanzada; en (9) $53 \%$ casos se encontraron ganglios positivos para metástasis; así, la supervivencia resulta muv reducida.

\section{CARCINOMA OF THE VULVA AT THE UNIVERSITY HOSPITAL}

\section{SUMMARY}

This study analizes 17 cases of cancer of the vulva at Valle University Hospital between 1964 and 1972 .

$70 \%$ of the women were above 50 years of age; most of them grand multipara of low socio-economic condition, and only three of them presented venereal disease. In 14 cases there was epidermoid cancer. Most of the cases were late diagnoses caused by late medical visits. The main symptom was pruriginose ulcer, with some association with obesity in 6 cases. In 10 cases the disease started in the labia minora and labia majora, and in 2 cases in the clitoris.

Treatment in 14 cases was radical vulvectomy and total vulvectomy in 3 cases.

\section{REFERENCIAS}

1. M. MAURICE ABITBOL, M.D. Carcinoma of the Vulva: Improvements in the surgical approach. Am. J. Obstet-Gynecol Volume 117 - Number 4. Obst. 1973.

2. COLLINS, C.G., ROMAN LOPEZ, J.J. and LEE. Am. J. Obst-Gynecol 108: 1187, 1971.

3. DAILY. J; KAPLAN, A.L. and KAUFMAN R.H: Obst-Gynecol 36: 845, 1970.

4. NIKOLAS A. JANOVSKI, CHARLES. P. DOUGLAS. Diseases of the Vulva.
Seven patients survived for more than five years.

Periodic follow-up visits were not possible because of lack of cooperation from the patients. Most cases were clinical studies II and III.

Early diagnosis is necessary in order to increase survival in this group of patients. Once the symptoms appeared, at least one year elapsed before those patients sought medical help and received adequate diagnosis and treatment.

The relationship between the size of the damaged tissue and metastasis is known. In most cases, the disease was advanced. 9 cases presented positive signs for metastasis making survival very difficult.

5. FRANKLIN E. W. and RUTLEDGE, F.D. Obst-Gynecol, 39: 165, 1972.

6. HAY, D.M. and COLEF. M. Am. J. ObstGynecol 108: 1187, 1971.

7. BARCLAY D.L., COLLINS C.G.,HANSEN L.H. Pacientes problema con Cáncer de Vulva. Am. J. Obst. Ginec, 84; 1135, 1962.

8. BREEN J.L; at ALL. Basal Cell Carcinoma of the Vulva. American Journal of Obstetrics and Gynecology aug. 1975. 